AperTO - Archivio Istituzionale Open Access dell'Università di Torino

\title{
On The Standardization Of The Photocatalytic Gas/Solid Tests
}

\section{This is a pre print version of the following article:}

Original Citation:

\section{Availability:}

This version is available http://hdl.handle.net/2318/153224

since 2016-01-10T17:28:30Z

Published version:

DOI:10.1515/ijcre-2012-0045

Terms of use:

Open Access

Anyone can freely access the full text of works made available as "Open Access". Works made available under a Creative Commons license can be used according to the terms and conditions of said license. Use of all other works requires consent of the right holder (author or publisher) if not exempted from copyright protection by the applicable law. 


\title{
Author Query Form
}

\section{AC on the Standardization of the Photocatalytic Gas/Solid Tests}

Article: IJCRE-2012-0045

$\begin{array}{lll}\text { Query No } & \text { Page No } & \text { column No } \\ \text { Q1 } & 1 & 1 \\ \text { Q2 } & 1 & 1 \\ & & \\ \text { Q3 } & 2 & 1 \\ \text { Q4 } & 15 & 2 \\ \text { Q5 } & 16 & 1 \\ \text { Q6 } & 16 & 2\end{array}$

\author{
Query \\ Please check and confirm whether the added details (author names and \\ affiliations) are correct. \\ All variables (single and multi letters) are made italics throughout the \\ article. Please check if this is ok. \\ Please check whether the short title is ok as typeset. \\ Please provide article title for all journal references. \\ Please provide publisher location and name for references [6] and [7]. \\ Please provide year of publication for references [17] and [18].
}




\section{AC on the Standardization of the Photocatalytic Gas/Solid Tests}

\section{Abstract}

5 The central problem for standardization of photocatalytic efficiency of whatever substrate on an illuminated catalyst is the rate evaluation. For gas/solid experiments different reactors, like batch or flow-through either continuous stirred-tank reactor (CSTR) or plug flow reactor

10 (PFR), could be envisaged. The basic equations governing these reactors and the rate expression for them are presented here. Experiments show that a CSTR configuration presents a lot of advantages for practical use, as any volume, any shape of catalyst and any flow of gas into 15 the reactor can possibly be used. A CSTR configuration is superior to the standardized PFR as the resistance to mass transfer can be reduced by inside forced ventilation. Consequently, it gives an evaluation of the photocatalytic rate more close to the actual surface one. The rate for CSTR

20 at steady state must be calculated as $r\left(C_{o}\right)=C_{o} F \eta /(1-\eta)$, where $\eta$ is the conversion.

Keywords: photocatalysis, standardization, ISO, CEN, nitric oxide

*Corresponding author: Claudio Minero, Department of Chemistry, University of Turin, Torino, Italy, E-mail: Elaudio.minero@unito.it

25 Andrea Bedini, Rockwood Pigments Torino, Torino, Italy; Department of Chemistry, University of Turin, Torino, Italy,

E-mail: andrea.bedini.to@gmail.com

Marco Minella, Department of Chemistry, University of Forino,

30

Torino, Italy, E-mail: marco.minella@unito.it

\section{Introduction}

The possible market for photocatalytic materials, in the form of powders, built-in powders, thin films and nanostructured materials, as well as devices directed to specific

35 applications, is estimated in exponential growth [1]. Commercial competition of the photocatalytic process for pollutant abatement is for now limited by the low photon efficiency that make expensive both solar plants (for the need of a large surface and investment capital) and artificial systems (for the need of power and high 40 running costs). However, niche application for air purification, deodorizing, anti-bacterial and anti-fungal properties, and mainly self-cleaning are taking large market interest. The competition will mainly be based on the relative performance of these new materials and 45 products.

The evaluation of performance is always related one way or another to the evaluation of the quantum yield, or of some less demanding related quantity like the photon efficiency. The key role is then played by the evaluation 50 of the chemical rate by which some standard compound is transformed, and the rate of photon absorption. Although the last can be in some instance simplified by fixing the spectral distribution of incoming light and its intensity $I_{o}$ (but not avoiding the scattering problem), 55 also the evaluation of the rate poses problems, mainly related, for the scope of standardization and laboratory evaluation, to what the macroscopic rate means, and to the devices (reactors) used for its evaluation. The rate of abatement (mole or grams per time, and eventually per 60 unit surface area) is the only quantity that is of some help to engineering, of relevance to application and also essential for basic research development.

The mechanistic (microscopic) kinetic analysis for photocatalytic reactions is quite complex, both for the 65 numerous essential primary reactions involved, and the number of different reactive species implicated (holes, adsorbed and free $\mathrm{OH}$ radical, $\mathrm{O}_{2-}, \mathrm{O}_{2}$, singlet oxygen...), to which chosen substrates are sensitive, leading to different evaluation of performance depending on their very 70 nature. The photocatalytic mechanism is by itself so complex that an exact kinetic analysis is prohibitive. For example, after the photo-excitation of the semiconductor when the primary oxidant species, $\mathrm{h}^{+}$reaches the surface, it can react with surface hydroxyl groups (trapped hole) 75 or molecular water [2]. The direct or mediated electron transfer influences the primary kinetic steps, the mechanism of oxidation and the probability of back-electron transfer. Since several factors influence the photon efficiency, including the reactant concentration, the possible 80 
adsorption on the active surface, the light absorbed and the reactor geometry (for its intimate relation to light distribution and scattering [3]), only an approximate kinetic analysis, based on the primary reactions taking

85 place at the surface of the photocatalyst, and on the possible back reactions (recombination reactions of charge carriers with primary radicals or species formed) is possible. This analysis pointed out that the primary bulk recombination of the photogenerated carriers, and

90 the back reactions [4], are responsible for the low quantum yields and the observed Langmuir-type or peaked shape of the rate vs. the substrate concentration [4]. It was demonstrated [5] that only under hypotheses that strongly simplify the system, analytical relationships for

95 the rate expressed in terms of parameters having physical significance are attainable.

Owing to all the above factors that contribute to a complex dependence of the rate on the substrate concentration, for the scope of standardization an a priori fixing 100 of conditions (like substrate concentration) could be open to question, since an optimal concentration reflecting the best catalyst performance does not exist. In the presence of a peaked behavior of the rate against substrate concentration, the maximum of which in turn depends on the

105 light intensity $I_{o}$ and catalyst-specific properties, the choice could be questionable as the proposed test, depending on the catalyst-specific properties, inevitably leads to improper discrimination among different samples.

The choice for a standardization test of substrate type

110 and concentration, and other experimental parameters, is to cope with the pollutant type, concentration and eventually conditions of illumination found in the application. From microscopic analysis it comes out that, given the catalyst and system conditions, the rate is at least a func-

115 tion $r\left(C_{o}, I_{o}\right)$, where $C_{o}$ is the input concentration of the pollutant in the reactor. As for devices directed to specific applications, their performance must be expressed as $r\left(C_{o}\right)$, leaving to the customer the evaluation of the (electric or light) power needed to attain a definite goal. For engi-

120 neering purposes, the rate must be expressed in absolute terms, such as the moles (or mass) of pollutant transformed per unit time and unit catalyst surface area (like $\mathrm{mg} \mathrm{h}^{-1} \mathrm{~m}^{-2}$ ), or for a device as the moles (or mass) of pollutant transformed per unit time ((like $\left.\mathrm{mg} \mathrm{h}^{-1}\right)$.

125 The central problem for standardization for gas/solid tests is the rate evaluation using different reactors like batch or flow-through either continuous stirred-tank reactor (CSTR) or plug flow reactor (PFR). Among the used, proposed or approved protocols of standardization, there

130 are different reactor configurations (plug [6] or continuous flow $[7,8]$ as well as batch reactors [9] or circulating fluidized bed [10] using different kinds of substrates like VOC [11], NO [6], mixture of $\mathrm{NO}+\mathrm{NO}_{2}[7,12], \mathrm{NO}_{2}$ [13], acetaldehyde [14, 15], toluene [16], formaldehyde [17] and methyl mercaptan [18]). The standardization test that 135 comes out of major experimental evidence for photocatalytic gas/solid reactions is that developed on NO abatement. Thus, this test is selected for the experiments of this work. The reaction is in principle quite simple as it involves a gas/solid reaction in the absence of system 140 interferences like those in solution, coming out from solution composition, and for slurries for the unmanageable scattering evaluation. The reaction mechanism was studied in several literature papers [19-28] and will not be discussed here, where the focus will be directed to the 145 development of the suitable reactor for the rate evaluation.

Since the time evolution of the substrate concentration generally (but not always) follows an apparent pseudo-first-order decay, this assumption will be used (and proved) to exemplify advantages and drawbacks of 150 different approaches (at fixed $C_{o}$ and $I_{o}$ when the performance of a catalyst is to be determined). The extension of the steady-state equations for a flow-through reactor in the case of any order reactions will also be given.

\section{Theory}

Figure 1 shows the schematics of a CSTR reactor (top) of volume $V_{r}$ and of a PFR (bottom). In the inlet of PFR the rate is higher than in the outlet because of the different local concentrations (rate gradient). To fix conditions of a plug flow (piston flow), the channel of the flow on the 160 sample must be thin, as no upstream or downstream mixing could be present. The ISO 22197-1 norm and further Parts $2-5$ specify test methods for the determination of the performance of materials that are typically flat by continuous exposure of a test piece of fixed size 165 $(100 \times 50 \mathrm{~mm})$ to a fixed substrate concentration in the gas phase under illumination in a PFR. The section of flow is fixed $(50 \times 5 \mathrm{~mm})$ by proper positioning the sample at $5 \mathrm{~mm}$ from the top window through which the ultraviolet light normally hits the sample. This con- 170 figuration is problematic for many specimens (like concrete, road pavements, bitumen...) for which a perfect cutting is not possible and for samples for which surface curvature is sometimes present. A reactor allowing arbitrary sample volume and surface, like CSTR, is more 175 promising for real application, provided that proper experimental setting is utilized. Typically, a PFR is more efficient than a CSTR of the same volume. 

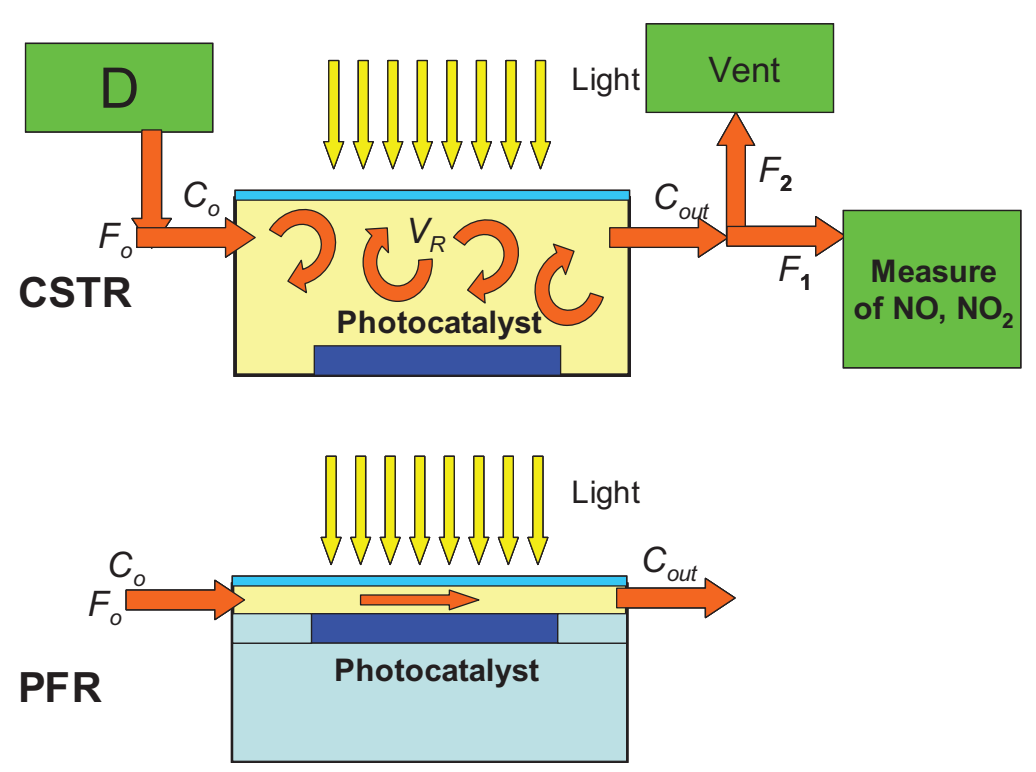

Figure 1 (Top): A schematic of a CSTR (well mixed) and (Bottom): of a PFR (piston flow). A flow $F_{o}$ enters at concentration $C_{o}$ and exits at $C_{o u t}$ after some test substrate molecules are consumed by photocatalytic reaction on the photocatalyst. Accessory configuration of the reactor is reported at top, where $D$ is a system for gas mixture supply/preparation (i.e. a diluter). The external lamp provides an illumination intensity $I_{0}$ on the sample surface. The sample/lamp configuration can be replaced by a device fit inside the reactor. The flows are not arbitrary for a flow-through configuration, as the input flow $F_{o}$ must exceed that needed by the detector $\left(F_{1}\right.$, e.g. a chemiluminescence's detector for $\left.\mathrm{NO}_{\mathrm{x}}\right)$. For batch experiments the input flow is null and, consequently, the flow needed by the measuring system must be null (e.g. using onsite spectrophotometric measurements). The typical reactor volume used in UNI 11238-1 [7] is 3 L, but it can be arbitrarily chosen as demonstrated here.

Conversely, in a CSTR all the catalyst surfaces are 180 exposed to the same (output) concentration [29]. This condition implies perfect mixing (gradientless operation) that can be experimentally accomplished with an internal fan, using (almost) an arbitrary reactor volume. If perfect mixing is achieved, i.e. the residence time is at least 10 185 times the mixing time, the arbitrary reactor volume is central as different samples can be fit in the reactor, avoiding limitations on the sample size, or difficult operations like sample cutting and positioning. This type of reactor can be used to measure small catalyst 190 samples as well as large devices (air conditioners, photocatalytic fans...) without changing the theory behind and the meaning of the measured quantities. The unique requirement that differentiates its application to catalyst samples and to devices is the external light source, which 195 is not needed in the latter case.

\subsection{Operation of stirred tank reactor (STR)}

The STR shown in Figure 1 (top) is normally operated

1. by purging it with air (or some other diluent gas if needed) without the pollutant under examination (NO, reactor form previous runs. At the end of this stage, the pollutant concentration in the reactor is null.

2. by subsequent filling with the pollutants at the proper concentration $C_{o}$ in the diluent gas under dark. Defining a normalized concentration $C^{*}=C / C_{o}$ and 205 a dimensionless time $t^{*}=t / t_{R}$, where $\left.t_{R}=\left(V_{R} / F\right)\right)$ is the reactor space time (residence time), the mass balance per unit time inside the reactor is

$$
\frac{\mathrm{d}}{\mathrm{d} t^{*}} C_{d a r k}^{*}=1-C_{d a r k}^{*}
$$

where $C_{d a r k}^{*}$ is the normalized concentration measured at 210 the output after exponential dilution inside the reactor. The straightforward solution under the initial condition that $C_{\text {dark }}^{*}(0)=0$ gives

$$
C_{d a r k}^{*}=1-\exp \left(-t^{*}\right)
$$

from which it is evident that the equilibration time 215 depends on the residence time $t_{R}$ (flow $F$ and the reactor volume $V_{R}$ ). The time evolution of the concentration inside the reactor during the filling operation is reported in Figure 2. In a CSTR with the sample under test inside it, if adsorption is present, the time required to recover 220 the input concentration is longer than that required without the sample (void reactor). This check will confirm that 


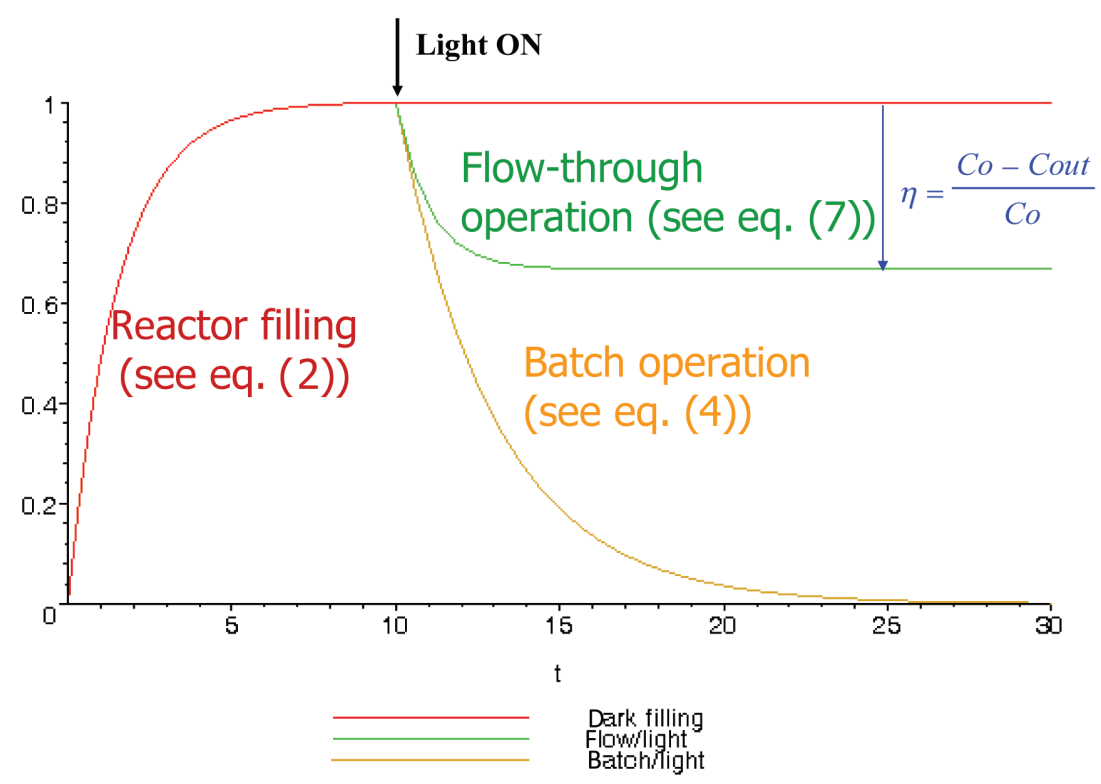

Figure 2 Normalized concentration $C^{*}$ inside a CSTR during dark filling and subsequent operation under light for a batch or flow-through condition.

adsorption is present. In the case of NO used in these tests, adsorption could be neglected.

As larger is the reactor volume, larger must be the used flow to avoid excessive equilibration times. The difficulty (and costs) to work with excessive $V_{R}$ is apparent, although somehow this is unavoidable, as for the performance assessment of bulky devices.

A PFR is operated in the same way, except that eqs [1] and [2] are not applicable. Given the small $V_{R}$, purging and filling times are shorter than in a CSTR.

After stages (1) and (2) for a STR, two ways of operation are then possible: (1) batch experiments; (2) flow-through operation. The time evolution of the concentration inside the reactor under ways of operation (1) and (2) after filling and light on is reported below and exemplified in Figure 2.

\subsubsection{Batch experiments}

240 After filling the reactor, the input flow is stopped, the reactor is closed and light is powered on. The evolution of the initial concentration of pollutant $C_{o}$ is measured as a function of time. This operation needs that (a) the reactor has no leaks, otherwise the pollutant concentra-

245 tion decreases without intervention of the photocatalytic reaction. The effect of leaks will be examined later; (b) the analyzer should consume as little as possible, or at best null volume of gas inside the reactor. This could be accomplished by onsite spectrophotometric measurements, as well as using a variety of sensors 250 (semiconductor-based, electrochemical...). If the pollutant has to be measured by a technique that needs sampling (as for $\mathrm{NO}_{x}$ the chemiluminescence's method, for VOC the GC...), the reactor volume must be large enough to assure that the sampled volume does not reduce sig- 255 nificantly the internal gas pressure (to avoid reverse leaks and improper operation of the detector).

Following a common choice in chemical reactor engineering, in the case under investigation the dimensionless Damköhler number is $D a=k_{R} S / F$. The mass balance 260 per unit time inside the reactor gives

$$
\frac{\mathrm{d}}{\mathrm{d} t^{*}} C_{b}^{*}=-D a C_{b}^{*}
$$

where $C^{*}{ }_{b}(t)$ is the normalized concentration inside the batch reactor (index $b$ ) that changes with time according to the photocatalytic reaction rate $-k_{R} S C_{b}(t)$, for which it 265 is assumed a first-order reaction rate and a specific rate constant $k_{R}$ (per unit area $S$ ). The rate of the photocatalytic reaction is in fact proportional to the surface concentration of the species $\{B\}$ that is related to the bulk concentration $[B]$ (here $C(t)$ ) by a proper isotherm [5]. For 270 gas-solid reactions and usual low concentrations, the adsorption could be assumed linear and the adsorption constant is collected in $k_{R}$. Other effects due to the local surface conditions (e.g. a resistance to the mass transfer due to the boundary layer) are also included in $k_{R}$. 
The straightforward solution of eq. [3] under the initial condition that $C_{b}^{\star}(0)=1$ gives

$$
C_{b}^{*}=\exp \left(-D a t^{*}\right)=\exp \left(-k_{\text {obs }} t\right)
$$

from which it is evident that the initial concentration $C_{o}$ 280 decays exponentially with $k_{o b s}=k_{R} S / V_{R}$, where $k_{R}$ is the linear photocatalytic intrinsic rate of degradation, $S$ is the sample surface and $V_{R}$ is the reactor volume. Accordingly, the rate normalized per surface area and extrapolated at $t=0$ to have $r\left(C_{\mathrm{o}}\right)=k_{R} C_{\mathrm{o}}$, for a batch 285 experiment is experimentally obtained by using eq. [5]:

$$
r_{b}\left(C_{0}\right)=\frac{C_{0} k_{o b s} V_{R}}{S}
$$

where the (net) volume of the reactor must be accounted for. The standardized protocols and good laboratory practice must explicitly fix that the rate must be calculated

290 using eq. [5]. The value of $k_{o b s}$ can be obtained from a fit on a set of data points $\left(C_{b}(t), t\right)$ using eq. [4] (or its log linearized form) and observing if the hypothesis of a firstorder reaction is obeyed, or, for practical and cost reasons, using a single point at time $t$ where $k_{o b s}=-\ln \left(C_{b}(t) / C_{o}\right) / t$.

295 This last procedure is more risky as statistical uncertainty is not assessed.

The drawbacks of the batch experiment are the extrapolation at $t=0$ (where $C=C_{o}$, using data at later times), and the deviations from the postulated law due to possi-

300 ble chemical mechanism changes during the reaction time, which are not directly evident, and reflect in a possible error in the evaluation of the initial rate.

Batch experiments are mandatory due to practical reasons for large volume reactors. This condition was

305 fixed in the AFNOR [12] norm. Because by using large reactors it is difficult to avoid leaks, $C_{o}$ is observed to decay in the dark. In this case it is necessary to measure in the dark the time evolution $C_{b}(t)$ as a function of time. A straightforward analysis shows that $C_{b, \text { dark }}(t)=C_{o}$ exp $310\left[-k_{\text {leak }} t\right]$, where $k_{\text {leak }}=F_{\text {leak }} / V_{R}$, and $F_{\text {leak }}$ is the outflow. When both the photocatalytic rate and the leaks are present, $C_{b}(t)=C_{o} \exp \left[-\left(k_{\text {leak }}+k_{o b s}\right) t\right]$. The final time evolution of $C(t)$ is then $C_{b}(t)=C_{b, \text { dark }}(t) \exp \left[-k_{o b s} t\right]$, which is similar to eq. [4], when $C_{o}$ is replaced with $C_{b, \text { dark }}(t)$. Note

315 that for the rate evaluation it is the ratio $C_{b}(t) / C_{b, \text { dark }}(t)$ that has to used, and not the conversion given by the difference $C_{b, \text { dark }}(t)-C_{b}(t)$ at a given time $t$.

\subsubsection{Flow-through experiments}

After filling the reactor, the input flow is maintained, and 320 light powered on. The output concentration of pollutant
$C_{\text {out }}$ is measured as a function of time. If the amount of pollutant consumed by the photocatalytic reaction (or other possible thermal and photolytic reactions) is constant over time, a stationary state is reached at which $C_{\text {out }}$ is constant.

The flow-through operation is in general recommended as less stringent experimental requirements are necessary. Owing to the action of internal fan that quickly mix the internal gas, this way of operation is compatible with additional downstream outflow, which simply 330 divide the output flow into different output channels. Thus, it is less stringent on reactor sealing, and it is compatible both with onsite and sampling measurement techniques. For the measurement by sampling, the requirement is that the flow used by the sampling tech- 335 nique would be lower than the input one. For assuring that leaks are admissible, the outflow of leaks must be negligible with respect to the flow generated by the fan and the flow eventually used by the sampling technique, and a small positive differential pressure must be present 340 between the inside reactor and outside. In addition, if the specific reactivity changes during the experiment time window, for example for catalyst poisoning, the effect could be easily realized as $C_{\text {out }}$ in a flow-through experiment will (slowly) change as a function of time. 345 Conversely, in a batch experiment (see above) the effect is convolute with the time evolution of $C(t)$ inside the reactor, and subsequent batch experiments on the same sample will show different decaying constants, or different initial rates (see above). This renders tricky the eva- 350 luation of $r\left(C_{o}, I_{o}\right)$ using batch experiments.

The mass balance per unit time inside the CSTR reactor is

$$
\frac{\mathrm{d}}{\mathrm{d} t^{*}} C_{f}^{*}=1-(1+D a) C_{f}^{*}
$$

where $C_{f}^{*}$ is the time-changing concentration inside the 355 flow-through reactor (index f) due to the photocatalytic reaction rate $-k_{R} S C_{f}(t)$ and exponential dilution. The meaning of other symbols is as above.

The flow-through or continuous flow experiments under condition of ideal mixing (CISRT) are described 360 as a function of time. The solution of eq. [6] under the initial condition that $C_{f}^{*}(0)=1$ is

$$
C_{f}^{*}=\frac{1+D a \exp \left(-(1+D a) t^{*}\right)}{(1+D a)}
$$

from which it is evident that two terms play a key role, depending on the relative weights of the residence 365 time $t_{R}=V_{R} / F$ (see also eq. [2]), and the photocatalytic 
rate (accounted for in the Damköhler number, see also eq. [3]).

Eq. [7] with the limit $t^{*}=0$ (when light is ON, and

370 the concentration decreases steeply, see Figure 2) could be used to find the value of $D a$ and then of $k_{R}$. Usually, the derivative of the initial concentration data is utilized to obtain the evaluation of the initial rate of reaction at the initial concentration $\mathrm{C}_{\mathrm{o}}$. From eq. [7] at first approx-

375 imation the initial rate is given by $\left(-d C_{f}^{*} / d t^{*}\right)_{t^{*} \rightarrow 0}=D a$. The rate normalized per surface area $r\left(C_{o}\right)=D a F C_{o} /$ $S=k_{R} C_{o}$ could then be calculated. However, often the decrease is so steep (depending on $t_{R}$, see below), that it is faster that the time response of the analyzer. This 380 procedure is in general to be avoided.

Eq. [7] shows that as $V_{R}$ is smaller, lesser is the time required to drop the time dependence of $C_{f}^{*}(t)$ and reach a constant value $C_{\infty}$ independent on the time and volume of the reactor (see the plateau in Figure 2). From eq. [7] as $385 t^{*} \rightarrow \infty$ (i.e. at steady state)

$$
C_{\infty}^{*}=\frac{1}{1+D a}
$$

At steady state the conversion $\eta=\left(1-C_{\text {out }}^{*}\right)$ is constant, and noting that $C_{\text {out }}=C_{\infty}$

$$
\eta_{\infty}=\frac{D a}{1+D a}
$$

390 From eqs. [8] and [9] it is evident that:

1. as the flow $F$ is very large (actually $D a \rightarrow 0$ ), $C_{\infty}^{*}=1$ or $\eta_{\infty}=0$. In this case some experimental difficulties could arise due to uncertainties in the measurement of small differences between $C_{\text {out }}$ and $C_{o}$.

2. the actual conversion $\eta_{\infty}$ or $C_{\text {out }}$ depends only on $D a$.

a. Given a specimen with definite photocatalytic reactivity $k_{R}$, the actual conversion depends on $F$ and the specimen area $\mathrm{S}$, that is on the experi-

The rate (at steady state) is normally calculated from experimental $\eta_{\infty}$ (see published standards) using the reasoning that rate $=-\Delta n / t_{R}$, where $-\Delta n$ is the amount (moles or mass) consumed in the "single pass" through the reactor, and thus rate $=-\Delta n / t_{R}=F\left(C_{o}-C_{\infty}\right)$. Given 415 the definition above of the conversion, it comes out that the rate normalized per surface area is

$$
r_{\infty}=\frac{C_{0} \eta_{\infty} F}{S}
$$

The rate evaluated from eq. [10] has the drawback cited above for eq. [9], and in particular, although $F$ is explicit 420 in eq. [10], the rate still depends on the experimental flow because $\eta_{\infty}$ depends on it. It is evident that $r_{\infty}=r\left(C_{\text {out }}\right)$, and thus that it reflects the experimental conditions used for its evaluation, not a property specific of the catalyst. This property is undoubtedly $k_{R}$. From eq. [9] it comes out 425 that $k_{R}=\eta_{\infty} F /\left(S\left(1-\eta_{\infty}\right)\right)$ and the rate $r\left(C_{o}\right)=k_{R} C_{o}$, is given by eq. [11]. Alternatively, from eq. [9] the value of $D a$ can be experimentally obtained as $D a=\eta_{\infty} /\left(1-\eta_{\infty}\right)$. It is then straightforward that the rate $r\left(C_{o}\right)=k_{R} C_{o}=D a F$ $C_{o} / S$ is expressed as:

$$
r_{\left(C_{o}\right)}=\frac{C_{0} \eta_{\infty} F}{S\left(1-\eta_{\infty}\right)}
$$

The rate $r\left(C_{\mathrm{o}}\right)$ given in eq. [11] should be independent of flow and experimentally determined $\eta_{\infty}$, which in turn depends on the flow and the specific reactivity. Using eq. [11] the rate is then evaluated at the input concentra- 435 tion and does not depend on the experimentally used flow. By comparing eq. [11] with eq. [10], a correction factor $1 /\left(1-\eta_{\infty}\right)$ is evident, which is quite relevant when $\eta_{\infty}$ is large. Thus, the often reported photocatalytic rates are underestimated. Eq. [11] is the main result of the 440 above treatment.

The above treatment is valid only for first-order reactions. When the photocatalytic rate in eq. [6] is not of first order, let us say $r_{\text {cat }}=k_{R} S C_{f}(t)^{n}$, where $n$ is the reaction order, the modified eq. [6] has analytical solutions for 445 definite values of reaction order $n$. As the solution of eq. [6] showed that the most interesting case is the steady state limit, setting $d C_{f}^{*} / d t^{*}=0$, a simple analytical equation for the rate is obtained.

$$
r_{n\left(C_{o}\right)}=\frac{C_{0} \eta_{\infty} F}{S\left(1-\eta_{\infty}\right)^{n}}
$$

This equation is a generalization of eq. [11] for any reaction order $\mathrm{n}$. However, the application of this last equation requires the knowledge of the reaction order. As it will be discussed later, $n$ can be obtained without the need to check the dependence of $r\left(C_{o}\right)$ from $C_{o}$, as this 455 will result in an impossible task as $n$ in the equation for calculating the rate is a priori unknown.

According to eqs. [11] or [12], all experiments made at different $(F, S)$ will give a different steady state 
460 conversion, but all must converge on a unique value for the rate. The experiments reported below will confirm the validity of eqs. [11] and [12].

\subsection{Operation of PFR}

The operation of a PFR is similar to a CSTR for the filling 465 and irradiation, except that basic mathematical description is different. Only the flow-through operation is possible. Here only a brief discussion is given for comparison purposes with that reported for a CSTR. A PFR under steady-state conditions is considered to simplify the treat-

470 ment, otherwise as time dependence is considered partial differential equations are involved. From the mass balance per unit time inside a section of the flow channel of thickness $\mathrm{d} x$

$$
\mathrm{d} C^{*}(x)=-C^{*}(x) \mathrm{d} D a
$$

475 where $C^{\star}(x)$ is the changing concentration inside the reactor as a function of position $x$ due to the photocatalytic reaction rate $-k_{R} S C^{*}(x)$. The meaning of other symbols is as above.

The solution of eq. [13] is quite simple:

$$
C_{f}^{*}=\exp (-D a)
$$

The conversion at steady state $\eta_{\infty}=\left(1-C_{\text {out }}^{*}\right)$ is

$$
\eta_{\infty}=1-\exp (-D a)
$$

and depends on the flow $F, S$ and $k_{R}$, but follows a relationship different from that of CSTR as compared to 485 eq. [9]. For this reason the ISO norm [6] properly set up the surface of the catalyst exposed to the flow, the flow $F$ and the height of the channel to ensure the plug flow. Da is easily found from eq. [15].

Coming back to the evaluation of the rate $r\left(C_{o}\right)=D a F$

$490 C_{o} / S$ as above, the rate normalized for the surface area, evaluated at the input concentration by a PFR for a firstorder reaction, is given by

$$
r_{\left(C_{o}\right)}=\frac{C_{0} F}{S} \ln \left(\frac{1}{1-\eta_{\infty}}\right)
$$

The rate $r\left(C_{o}\right)$ given in eq. [16] should be independent of 495 flow and experimentally determined $\eta_{\infty}$, which in turn depends on flow and the specific reactivity. Note that only under the limit that $n \rightarrow 0, r\left(C_{o}\right)$ is given by eq. [10], or at first order when $\eta \ll 1$, using the correspondence limit $(\ln (w), w \rightarrow 0)=w-1$, by eq. [11]. Contrary 500 to the case of CSTR for which eq. [11], obtained for a CSTR, is valid for all the range $0 \leq \eta \leq 1$, when it is applied to a PFR in substitution of the correct eq. [16], eq. [11] gives rise to errors as for the PFR case it is just an approximation. The error is acceptable only when the conversion is very low, that is when for a differential 505 reactor it is possible to assume an almost constant reactant concentration throughout the reactor. An excess error of $20 \%$ occurs using eq. [11] at $\eta=0.3$. Much worse is the case with eq. [10]. Thus, the use of eq. [10] must be definitely deprecated for both reactor types. Its 510 use is just a wrong practice, which should be avoided.

\section{Experimental}

The experiments have been carried out in a CSTR with fan using a photocatalytic specimen with high conversion, as to prove eq. [11] and for differentiating with eq. [10], $\eta_{\infty} 515$ must be large. For this purpose, films of the catalyst were prepared using two different experimental procedures: (A) A slurry was prepared by mixing $8 \mathrm{~g}$ of $\mathrm{TiO}_{2} \mathrm{P} 25$ washed catalyst into $200 \mathrm{ml}$ of ultrapure water and sonicated for $30 \mathrm{~min}$. These slurries were deposited manually 520 over a $10 \times 10 \mathrm{~cm}$ glass slab and then dried in oven at $60^{\circ} \mathrm{C}$ for three hours to have a final loading of $20 \mathrm{~g} \mathrm{~m}^{-2}$. Experiments with this catalyst are reported as exp.\#1. (B) A slurry was prepared by mixing $8 \mathrm{~g}$ of $\mathrm{TiO}_{2} \mathrm{P} 25$ washed catalyst into a solution of $30 \mathrm{ml}$ of $\mathrm{NH}_{3}+1 \mathrm{ml} 525$ of acetylacetone $+15 \mathrm{ml}$ Igepal CO-720 (Aldrich). This slurry was brought to a final volume of $200 \mathrm{ml}$ by addition of ultrapure water, and then sonicated for 30 min. A better dispersion was obtained compared to the case A). The slurry was then deposited manually 530 over a $10 \times 10 \mathrm{~cm}$ glass slab to obtain a final loading of $20 \mathrm{~g} \mathrm{~m}^{-2}$ as before, dried and fired in oven at $400^{\circ} \mathrm{C}$ for two hours to oxidize the organic carbon. It was observed that during the degradation experiments the catalyst was poisoned by side products. Then the cata- 535 lyst specimen was cleaned by heating it at $300^{\circ} \mathrm{C}$ for $60 \mathrm{~min}$ after each irradiation run. This procedure proved to recover the initial catalyst activity under the same conditions. Two catalyst specimens have been prepared, and experiments with these are reported as 540 exp.\#2 and exp.\#3. Following the same procedure, a sample of $10 \times 5 \mathrm{~cm}$ was prepared for tests in the ISO PFR reactor.

The photocatalytic tests were carried out using a home-built reactor, with walls ( $15 \mathrm{~mm}$ thick) and internal 545 supports in PMMA. The reactor has internal size of $160 \times 220 \times 85 \mathrm{~mm}(\mathrm{l} \times \mathrm{w} \times \mathrm{h})$. The reactor was equipped with a fan $\left(6 \times 6 \mathrm{~cm}\right.$ size, $12 \mathrm{~V}$, flow of $\left.27 \mathrm{~m}^{3} \mathrm{~h}^{-1}\right)$, a diffuser plate to ensure correct input of the inlet flow, 
550 and two lateral walls inside the box to allow return path of the inside flow to the fan, positioned at $2 \mathrm{~cm}$ apart from external walls. The reactor is equipped with a DeltaOhm thermohygrometer model HD 2101.2 to measure temperature and humidity during the tests. Moisture

555 inside the reactor chamber is provided through bubbling $\mathrm{N}_{2}$ flow into a humidifier (thermostated at $60^{\circ} \mathrm{C}$ containing water and saturated aqueous vapor), and regulated by changing the gas pressure of the $\mathrm{N}_{2}$ line before the mass flow controller. For tests under ISO PFR condition,

560 the ISO reactor suggested in the norm ISO 22197-1 [6] was adopted.

The experiments have been carried out using as substrate NO, as for the ISO test [6] the reaction gas mixture at proper concentrations was prepared using a Entech

565 dynamic diluter model 4600A, mixing $\mathrm{N}_{2}, \mathrm{O}_{2}$ and a standard of NO at $250 \mathrm{ppmv}$, previously prepared in a canister of $3 \mathrm{~L}$ volume at $3 \mathrm{~atm}$., using mass flow controllers with $5 \mathrm{~L} / \mathrm{min}, 1 \mathrm{~L} / \mathrm{min}$ and $10 \mathrm{cc} / \mathrm{min}$ maximum flow rates, respectively. Pure $\mathrm{N}_{2}, \mathrm{O}_{2}$ and $\mathrm{NO}$ gases were purchased 570 from SIAD (Italy). $\mathrm{NO}$ and $\mathrm{NO}_{2}$ were monitored through a Horiba monitor APNA 370 equipped with a chemiluminescence's detector. The irradiation was made by two Philips PL-S 9W/2P BLB lamps positioned a $5 \mathrm{~cm}$ center-to-center distance, having the emission band centered 575 at $368 \mathrm{~nm}$. The light intensity incident on the sample surface was measured with a Cofomegra power meter, and regulated at $20 \mathrm{~W} \mathrm{~m}^{-2}$ by adjusting the distance of the lamp from the sample.
The photocatalytic tests are then performed using the above catalyst placed in the CSTR reactor (net inside 580 volume $2.85 \mathrm{~L}$, see below) closed on the top with a Pyrex glass, and using NO (0.55 ppm in exp.\#1, $0.454 \mathrm{ppm}$ in exp.\#2, $0.487 \mathrm{ppm}$ in exp.\#3, $0.507 \mathrm{ppm}$ in PFR experiment), $\mathrm{NO}_{2}<5 \% \mathrm{NO}$, total gas flow $3 \mathrm{~L} / \mathrm{min}$ (unless otherwise indicated), relative humidity of $30 \%$, at 585 a temperature of $298 \mathrm{~K}$ and incident light intensity $20 \mathrm{~W} \mathrm{~m}^{-2}$. The tests on the dependence of the conversion from the CSTR volume have been carried out changing the net reactor volume by filling the reactor with PMMA blocks.

The effect of the fan speed in CSTR, operating the fan at different voltages, is reported in Figure 3. With lower fan speeds, the conversion is lower (or the output concentration is higher) not only because possible preferential paths from the input channel to the output are 595 possible, but mainly because a thicker boundary layer on the sample is formed, increasing mass transfer resistance, as will be discussed below. Mass transfer limitation were recently demonstrated also for slurries [30].

The effect of the boundary layer thickness is unavoid- 600 able even for very large reactor internal flows. It can be reduced using very high volumetric flow inside the reactor. Figure 3 inset reports the reciprocal conversion as a function of the inverse of the flow generated by the fan. The extrapolation to $1 / F \rightarrow 0$ implies the reduction of the 605 boundary layer to zero, and gives the maximum attainable conversion $\eta_{\infty, \max }\left(\eta_{\infty, \max }=0.81\right.$ under actual

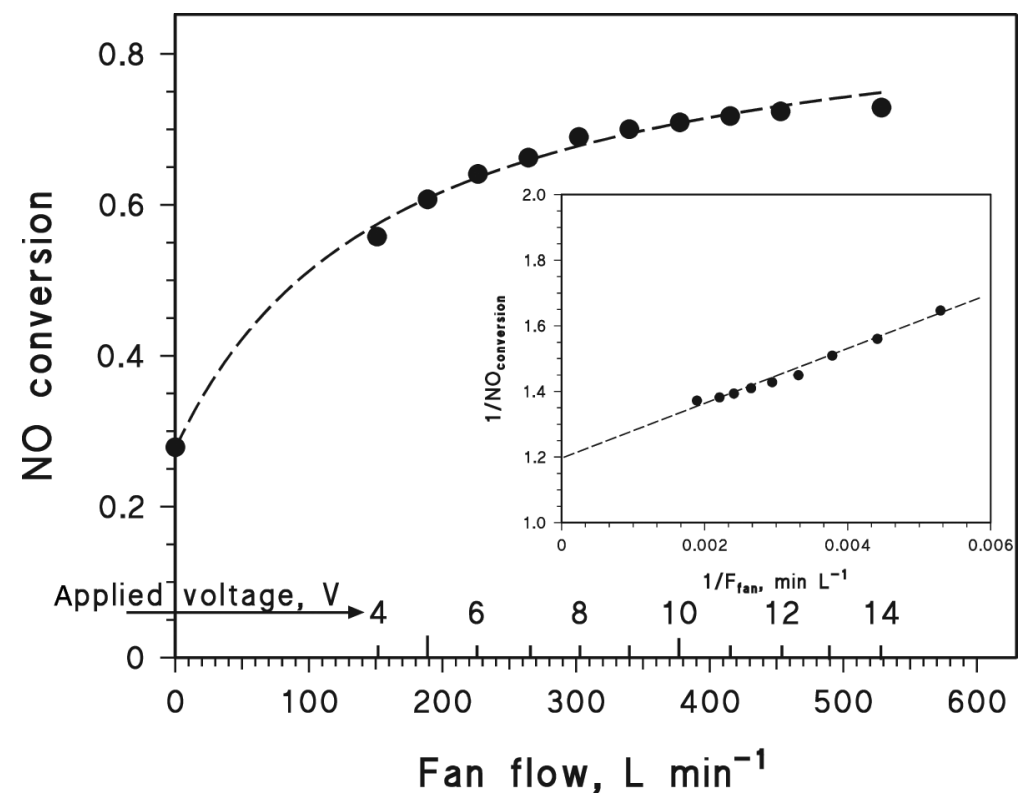

Figure 3 Conversion at steady state measured at different fan speeds, obtained by changing the CC voltage of the power supply of the fan. Inset: The reciprocal of the conversion as a function of the reciprocal flow. Data reported for catalyst \#2. 
experimental conditions for the sample used). By comparing the conversion at the nominal voltage of the fan $610\left(12 \mathrm{~V}, \eta_{\infty, \text { reactor }}=0.72\right)$ with the extrapolated $\eta_{\infty, \max }$, the maximum difference of conversion from the value corresponding to the absence of the boundary layer is just about $9 \%$. However, this extrapolated condition is never reached in practical applications. Thus, the conversion 615 values at steady state of Figure 3 (above $10 \mathrm{~V}$ for the actual fan, around $400-500 \mathrm{~L} \mathrm{~min}^{-1}$ of air flow generated by the fan inside the actual reactor) could be acceptable for a reasonable estimated rate. Under these conditions the experiment is also not critical as regards to the stabi-

620 lity of the flow (as the fan speed uncertainty implies a difference in few percent on conversion). The following reported experiments have been carried out under this condition.

\section{Results and discussion}

625 A typical experiment in which the NO concentration is monitored as function of time during filling in a CSTR, and after a constant value is reached, the illumination is started, as reported in Figure 4.

Different flows (1.9-4.5 $\mathrm{L} \mathrm{min}^{-1}$ ) have been used in 630 the experiments with the different prepared catalysts. It is evident that both in the filling region (as eq. [2] foresee) and at steady state (as eq. [8] foresees), the output concentration depends on the flow. Let us analyze the experimental obtained dependence of the concentration from time and flow $F$ in these two regions to assess the 635 validity of the developed model and the proper operation of the reactor.

\subsection{Filling region}

The NO concentration data collected in the filling region are linearized according to eq. [2] in Figure 5. The plot of 640 $\log \left(1-C_{\text {dark }}(t) / C_{o}\right)$ as a function of time must give a null intercept and a slope $=-1 / t_{R}=-F / V_{R}$. The actual intercept is non-null as the time scale is in arbitrary units. However, the data fit on a straight line with excellent correlation coefficients: 0.999 (2.0), 0.999 (2.5), 0.999645 (3.0), 0.994 (4.0), 0.998 (5.0), where in parentheses the actual flow $F$ is reported.

Because the slope $=-F / V_{R}$, a plot of the slopes obtained from fits on Figure 5 as function of $F$ will give a null intercept and a slope $=-1 / V_{R}$, from which 650 the net reactor volume could be estimated. This plot is reported in Figure 6. As the model predicts, the line is straight, with a correlation coefficient 0.987 . The value of the net reactor volume obtained, $V_{R}=2.83 \pm 0.05$, is in perfect agreement with that calculated from geo- 655 metric measurements of the reactor. This proves the theory based on the well-mixed conditions and that this very condition is verified by the experimental configuration.

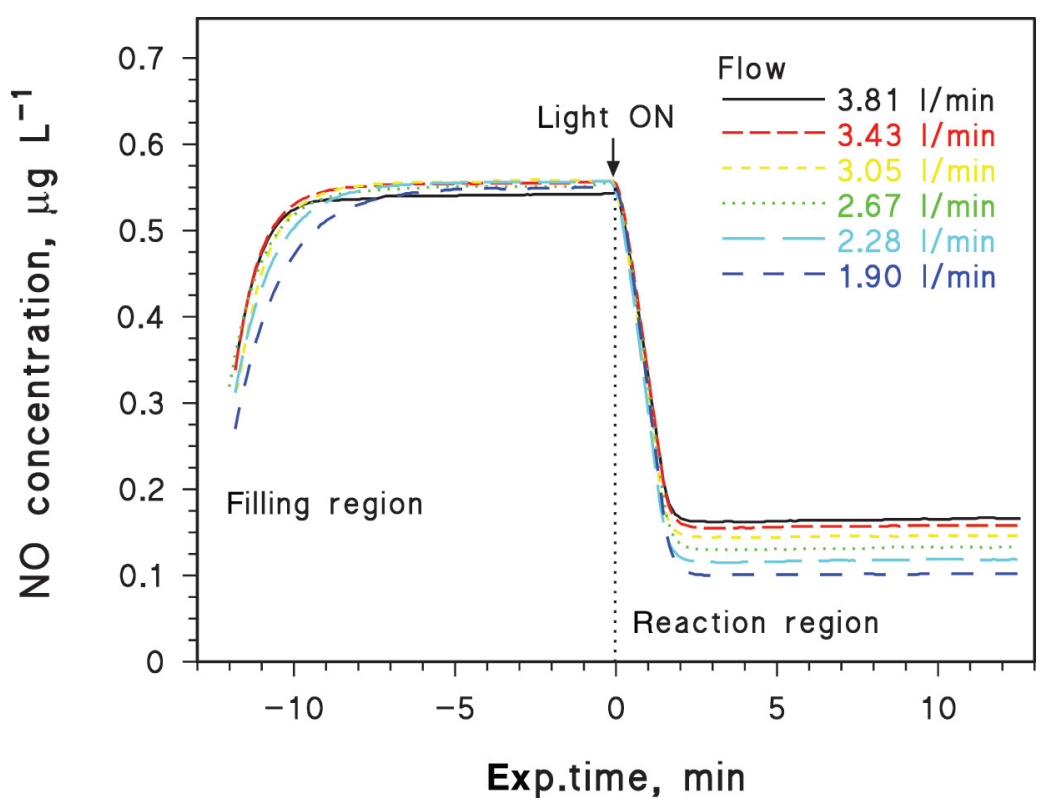

Figure 4 Time evolution of the NO concentration as a function of different flows into the reactor during filling, and after a constant value is reached, the illumination (data refer to exp.\#2). 


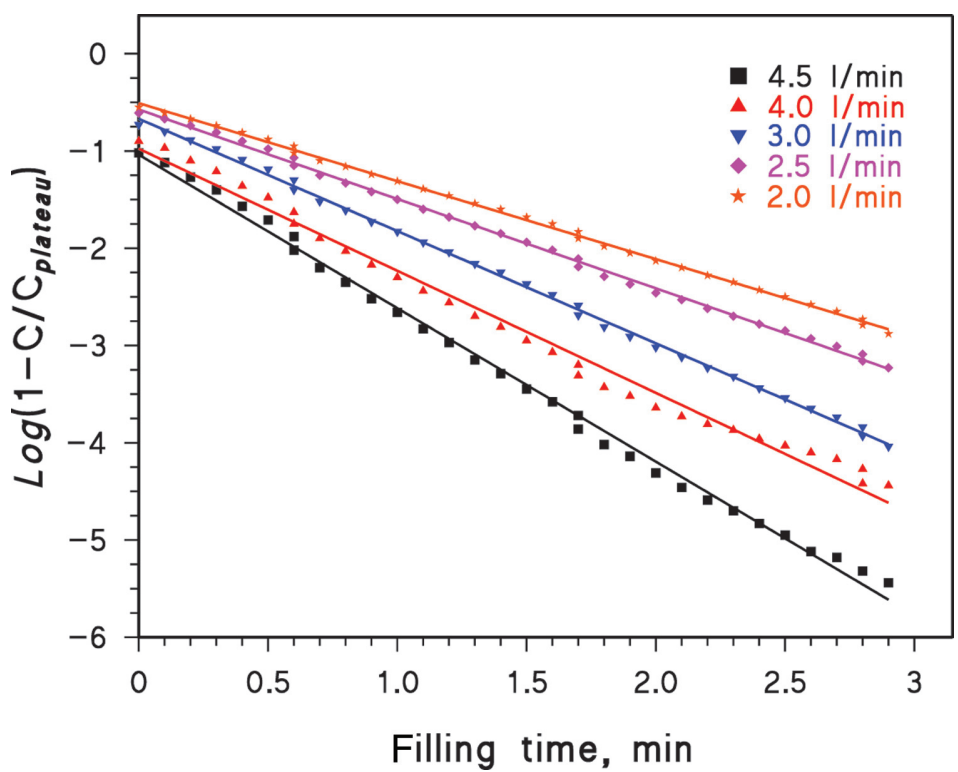

Figure 5 Linearization of NO concentration data in the filling region according to eq. [2] for exp.\#1. Similar trends are obtained in exp.\#2 and \#3 because the texture of the catalyst is not influential in the filling region.

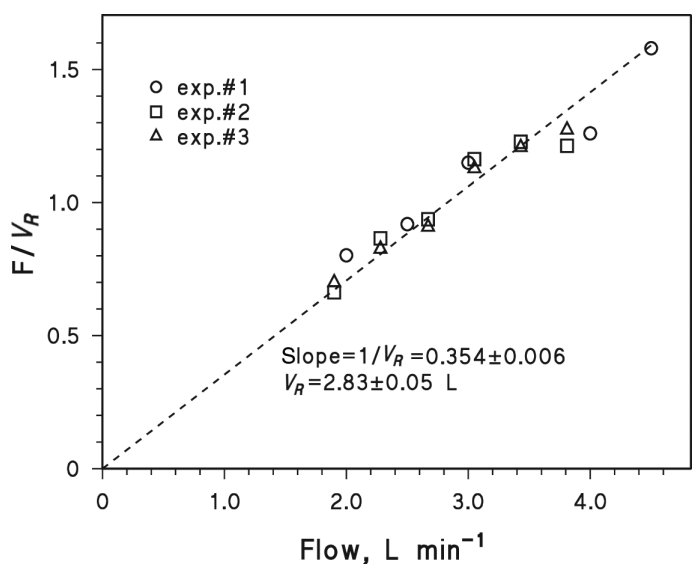

Figure 6 The slopes of straight lines obtained by linearization of eq. [2] (like those reported in Figure 5 for exp.\#1) as a function of the flow $F$. The inverse of the slope obtained is the reactor net volume.

\subsection{Reaction region}

The raw concentration data at steady state reported in Figure 4 are transformed into conversion in Figure 7.

For the concentration data, the conversion is higher as the flow is lower. It is expected that when the flow is 665 infinity, the conversion is null, and consequently the output concentration equals the input one $\left(C_{\text {out }}=C_{o}\right)$. Conversely, when the flow $\rightarrow 0$, the conversion will tend to 1 , as the residence time becomes infinite.

For the dependence on the reactor volume, eq. [9] 670 predicts that at steady state, the conversion is independent of the reactor volume. An experimental confirmation is reported in Figure 8. From this outcome it is

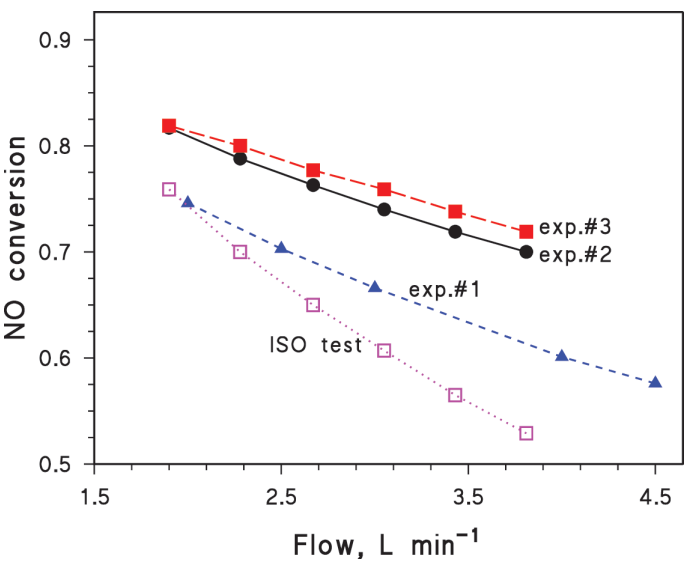

Figure 7 NO conversion at steady state $\eta_{\infty}=\left(C_{o}-C_{\text {out }}\right) / C_{o}$ in the reaction region as a function of the flow $F$ for the set of experiments \#1-3 in CSTR and for PFR (ISO) test using the same catalyst as in experiments \#2 or \#3.

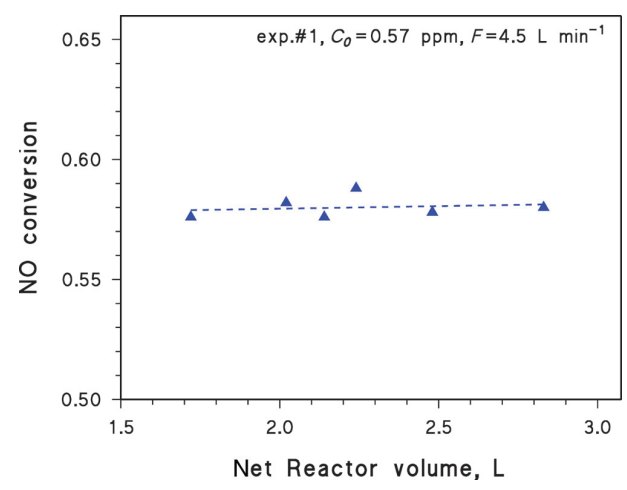

Figure 8 Dependence of the conversion at steady state from the reactor volume for the CSTR. Conditions are given in the figure. 
evident that also the rate, whatever the way of its calculation, is independent of the reactor volume. This con-

675 firms the theory and assures that any reactor volume is allowed, or at fixed reactor volume, any sample volume is allowed inside it, without changing the result of measurement.

\subsubsection{Reaction order}

Before using eq. [11], the reaction order must be verified, 680 as eq. [11] is valid for a first-order reaction, or eq. [12] requires in any case the knowledge of reaction order $n$. Remembering the definition of $D a$, and rewriting eq. [9] as

$$
\left(\frac{1}{\eta_{i}}-1\right)=\frac{F_{i}}{k_{R} S}
$$

the plot of $\left(1 / \eta_{i}-1\right)$ vs. the corresponding flow $F$ must give 685 a straight line with null intercept and slope $1 / k_{R} S$ only if the reaction is first order. This plot is reported in Figure 9.

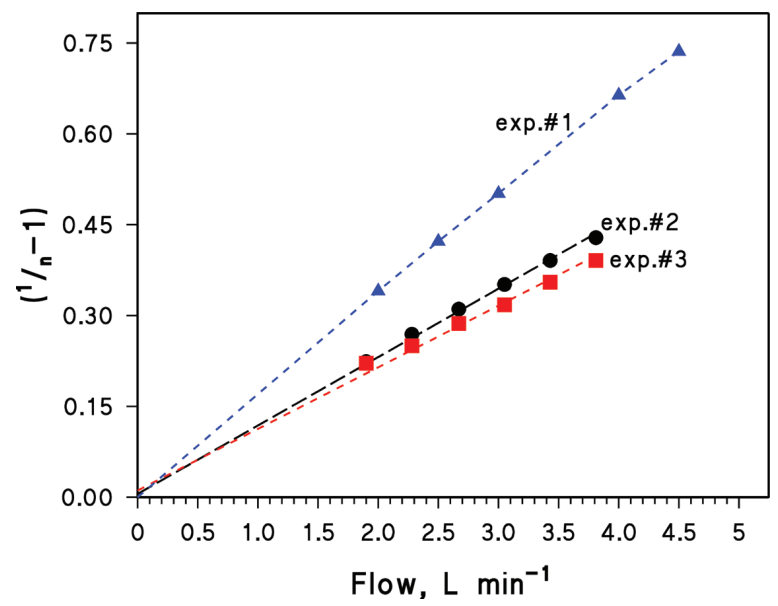

Figure 9 Test of reaction order using eq. [17a] for data of conversion obtained at different flows $F$.

An excellent straight line is obtained with null intercept and a superb correlation coefficient $(0.999$ for the three experiments). The resulting good correlation deriving from

690 theory proves: (a) again that the above theory is self-consistent with the experiment, (b) that, at least at [NO] near $0.5 \mathrm{ppm}$ the reaction rate is first order. Note that the proof was obtained at a fixed [NO] concentration changing the flow $F$. From the slope, the value of the rate constant 695 specific to the sample $k_{R} S$ can be obtained. The different catalysts show different reactivity, markedly exp.\#1 is quite different from the other two. The preparation procedure $B$ gives more active catalyst. The investigation of the cause is outside the scope of the present work.
A further demonstration of the first-order kinetic for 700 transformation of $\mathrm{NO}$ is reported at fixed flow by changing $[\mathrm{NO}]$ in Figure 10.

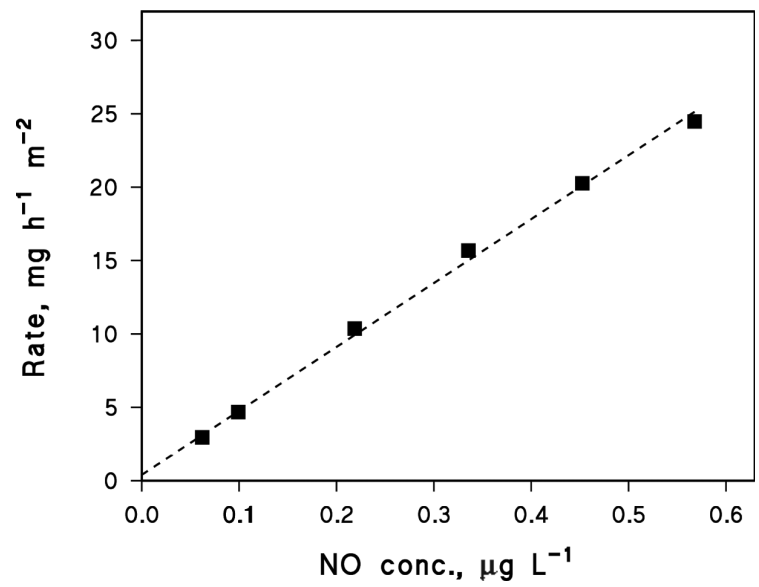

Figure 10 Dependence of the rate on NO concentration. Conditions: specimen \#1, $F=4.5 \mathrm{~L} \mathrm{~min}^{-1}$, rate calculated assuming first order using eq. [11].

Following the above reasoning, when the reaction is of not first order, the reaction order can be evaluated at fixed $C_{o}$ changing the flow $F$. Noting that, besides eq. [12], $r_{n}\left(C_{o}\right)=705$ $k_{R} C_{0}{ }^{n}$. It follows that $k_{R}=\left(C_{o}^{(1-n)} F \eta_{\infty} /\left(S\left(1-\eta_{\infty}\right)^{n}\right)\right.$. This result can be rewritten as

$$
\log \left(\eta_{i} F_{i}\right)=a+n \log \left(1-\eta_{i}\right)
$$

where $a$ is a constant at fixed input concentration, $a=\log \left(k_{R} S C_{o}{ }^{(n-1)}\right)$. Eq. [17b] is corresponding to eq. [17a] 710 when $n=1$. Using eq. [17b] the reaction order can be found as the slope of a plot of $\log \left(\eta_{i} F_{i}\right)$ vs. the corresponding $\log \left(1-\eta_{i}\right)$. After that the reaction order is known, and eq. [12] can be used to calculate the normalized rate $r\left(C_{o}\right)$.

\subsection{Rate evaluation}

Given that for NO transformation the reaction order is $n=1$, the normalized rate $r\left(C_{o}\right)$ could be calculated using eq. [11] for the CSTR and eq. [16] for the PFR. The results for the different flows and different catalyst specimens are reported in Figure 11. To account for the 720 different initial concentration $C_{o}$ and knowing that the kinetic is of first order, a normalized value for the rate was calculated assuming $C_{o}=0.50 \mathrm{ppm}$ for all the experiments. It is evident that eq. [10] fails (open symbols), as the rate depends on the experimental flow 725 casually used in the reactor as already discussed under paragraph 2.2. T4he experimental results confirm 


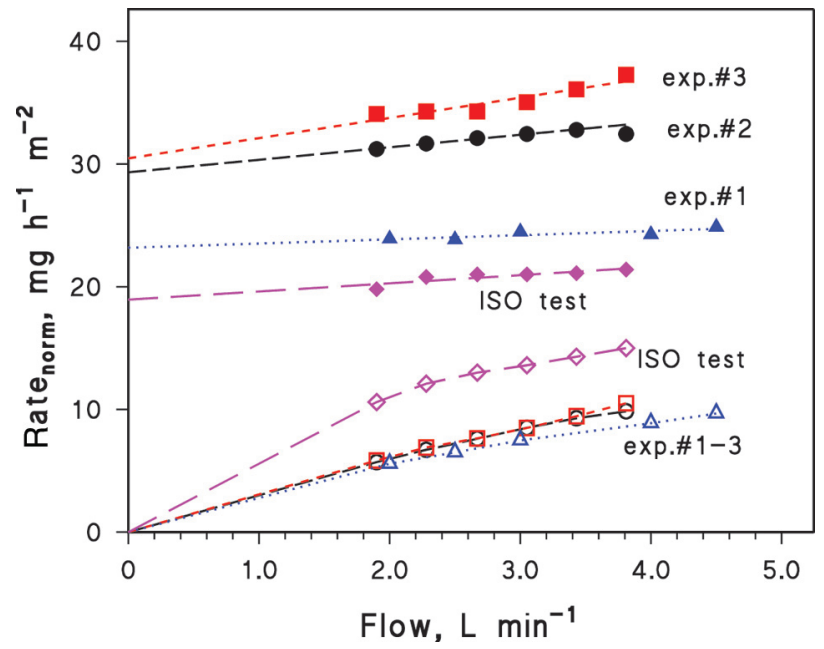

Figure 11 The rate normalized for input concentration (at $0.50 \mathrm{ppm}$ ) and surface area evaluated at different flow $F$ for the CSTR as calculated from eq. [10] (open symbols) and eq. [11] (full symbols), and for the PFR as calculated from eq. [10] (open symbols) and eq. [16] (full symbols) for the set of experiments.

that the use of eq. [10] is fully inconsistent for both reactors. Conversely, eq. [11] and eq. [16] for any used

730 flow, give (almost) the same value for the normalized rate. Thus, the results confirm the validity and usefulness of these equations. As a consequence, the reactors can be run at any flow without giving the resultant rate dependent on it. In conclusion, the rate can be evalu-

735 ated using eqs (11) and (12) for a CSTR or eq. [16] for a PFR in absolute terms, such as the number of moles (or mass) of pollutant transformed per unit time and unit catalyst surface area (like $\mathrm{mg} \mathrm{h}^{-1} \mathrm{~m}^{-2}$ ). For a CSTR used to assess the performance of a device, the rate is given 740 as the number of moles (or mass) of pollutant transformed per unit time (like $\mathrm{mg} \mathrm{h}^{-1}$ ).

\subsection{Effect of external pressure drop}

There is still a weak dependence on the flow, as the flow increase causes a positive increase in the rate. The rate

745 does not increase in exp.\#1, but for \#2 and \#3 the catalyst activity decreased by side-product formation on the catalyst as successive runs with increasing flow have been performed. A slow increase with the input flow is observed also for PFR (ISO test). This effect is merely

750 due to the counter-pressure made by connection holes and tubes at the outlet of the reactor (see Figure 1, top). In particular, the vent tube must have some length so as to avoid a back flow in the detector. Since the rate $r\left(C_{o}\right)$ will reflect change in $C_{o}$, which for a gas depends on pressure $\left(C_{o}=n / V=P / R T\right)$, this effect can be quantified 755 using the Poiseuille law for compressible fluids under isothermal conditions:

$$
\left(\frac{P_{i}}{P_{o}}\right)^{2}=1+\frac{16 F \mu L}{P_{o} \pi R^{4}}
$$

where $P_{i}$ is the input (vent) tube pressure (here the pressure sensed in the reactor), $P_{o}$ is the atmospheric outlet 760 pressure, $F$ is the volumetric flow, $\mu$ is the viscosity, $L$ is the length of the vent tube and $R$ its radius. Under firstorder conditions $\left(r\left(C_{i}\right) / r\left(C_{o}\right)\right)^{2}=\left(P_{i} / P_{o}\right)^{2}=1+\alpha F$. The rate increases linearly with the increasing flow as reported in Figure 11. From the Figure 11 data, averaged $\alpha$ result is 765 0.086 , a value compatible with the actual length and diameter of the vent tube $(4 \mathrm{~m} \times 2 \mathrm{~mm}$ id + connection holes). This very particular must also be fixed in the standardized protocols, although it is never cited. To reduce as much as possible the residual dependence of 770 the rate from the flow, the pressure drop must be reduced as much as possible by using large connection holes and vent tube internal diameter.

Taking the above precautions, the constancy of the calculated rate vs. the used flow opens new opportunities 775 for the reactor design (any volume, any shape, any flow), provided that the internal fluid is well mixed. This is quite an important conclusion for standardization and basic rate evaluation, as the sample can be of any shape, and often it is unpractical to cut it in the required 780 size and shape. Conversely, an ideal-mixed reactor conceived to accept most of the sample geometries encountered in practice has a large application.

The formula (16) also shows that conversion data obtained in the PFR (ISO test) are correctly calculated, 785 as they are invariant with the flow. As a consequence, eq. [16] must be used to report data from PFRs instead of the conversion, or the rate calculated using eq. [10] as it is valid only for very low conversions (see discussion below eq. [16] in Section 2.2).

\subsection{CSTR vS. PFR}

The difference observed for CSTR in experiments \#2 and \#3 with respect to PFR using the same catalyst should be discussed as it is surely outside the experimental error. It is not an effect of the surface area exposed to the flow, as 795 the rate is normalized for it according to eqs. [11] and [16]. As for the fan effect on the conversion of the CSTR already considered before, the cause could be related to the boundary layer formed near the catalyst surface. The 
800 tangential flow on the surface is quite different in the case of CSTR and PFR.

Boundary layers may be either laminar (layered), or turbulent (disordered) depending on the value of the Reynolds number [31]. For a plate on which the flow 805 is tangential, the critical $R e=5 \times 10^{5}$. For lower Reynolds numbers, the boundary layer is laminar and the streamwise velocity changes uniformly as one moves away from the wall. In this case the thickness of the boundary layer is $\delta=5 x / \sqrt{ } \operatorname{Re}(x)$. For higher Reynolds numbers, the bound-

810 ary layer is turbulent and the streamwise velocity is characterized by unsteady (changing with time) swirling flows inside the boundary layer. In this case the thickness of the boundary layer is $\delta=0.16 x /(\operatorname{Re}(x))^{1 / 7}$.

The Reynolds number is defined as $\operatorname{Re}(x)=x U / \mu$, 815 where $x$ for a plate is the distance from the leading edge, $U$ is the linear velocity and $\mu$ is the kinematic viscosity of the fluid (air). $U$ is related to the tangential flow $F$ by $U=F /$ $A$, where $A$ is the section of the reactor normal to the flow.

In the case of two reactors discussed, for the present 820 CSTR, the area section of the duct $A=16 \mathrm{~cm} \times 8.5 \mathrm{~cm}=$ $1.36 \times 10^{-2} \mathrm{~m}^{2}$, and for PFR (ISO) $\mathrm{A}=5 \mathrm{~cm} \times 0.5 \mathrm{~cm}=$ $2.5 \times 10^{-4} \mathrm{~m}^{2}$. As for CSTR $F_{\text {fan }}=500 \mathrm{~L} / \mathrm{min}=8.33 \times 10^{-3}$ $\mathrm{m}^{3} \mathrm{~s}^{-1}$, and for PFR for a typical inlet flow of $3 \mathrm{~L} \mathrm{~min}^{-1}=$ $5 \times 10^{-5} \mathrm{~m}^{3} \mathrm{~s}^{-1}$, the corresponding linear velocities based 825 on the actual cross-sectional area of the duct are $U_{C S R T}=0.61 \mathrm{~m} \mathrm{~s}^{-1}$ (neglecting back flow by reflection on opposite walls, minimized by lateral return channels) and $U_{P F R}=0.2 \mathrm{~m} \mathrm{~s}^{-1}$, respectively. These linear velocities are compared to the wind speed normally found in urban

830 air due to regional winds. In the case of traffic, local road air velocity could be much larger. This attests that (a) the tests are near ambient air conditions; (b) the conditions used in CSTR (see also Figure 3) are more close to real conditions. For the above linear velocities, the Reynold 835 number is $R e \ll<2,300$ for both reactors. However, due to the effect of the fan, inside the CSTR the flow is turbulent, while in ISO PFR the flow is definitely laminar.

Considering the boundary layer over a flat plate (as it is the photocatalyst sample), the average $R e$ on the cata840 lyst length $L$ used $(10 \mathrm{~cm})$ is

$$
\operatorname{Re}_{\text {ave }}=\frac{1}{L} \int_{0}^{L} \frac{x U}{\mu} d x=\frac{U L}{2 \mu}
$$

For the reactors considered, this implies $R e_{\text {ave,CSTR }}=1,820$ (not considering swirling flows inside the boundary layer induced by the fan) and $R e_{a v e, P F R}=600$. These numbers 845 confirm that the boundary layer flow is laminar. Under this condition, neglecting the fluid compressibility for the small pressure change in the reactors, the average boundary layer thickness is given by the average on the Blasius solution

$$
\delta_{\text {ave }}=\frac{1}{L} \int_{0}^{L} \frac{5 x}{\sqrt{\operatorname{Re}(x)}} d x=\frac{10}{3} \sqrt{\frac{\mu L}{U}}
$$

For the reactors considered, calculated $\delta_{\text {ave,CSTR }}=5.5 \mathrm{~mm}$ (actually less for the effect of fan), and $\delta_{a v e, P F R}=9.6 \mathrm{~mm}$. These numbers are quite large compared to the catalyst roughness (about $1 \mu \mathrm{m}$ ) and suggest that the model for the ISO PFR is unsuitable, as the $\delta_{\text {ave }}$ is larger than the 855 flow channel. Instead of a turbulent PFR, the ISO PFR is a laminar flow reactor maintaining the condition of no upstream or downstream mixing.

If the flow near the surface is laminar, the substrate molecules can reach the surface only by diffusion. 860 Assuming that diffusion from the bulk air to the surface is the limiting rate, the measured rate must be proportional to this flux $J$, given at first approximation by the Fick law $J=-D d c / d x$, where $d x$ could be assumed equal to the average boundary layer thickness, and $d c=C_{o}$, assuming 865 at the surface an almost zero substrate concentration:

$$
\text { rate }=k^{\prime} \frac{\text { Co }}{\delta_{\text {ave }}}
$$

Considering the above calculated $\delta_{\text {ave }}$, this equation calculates that rate $_{C S T R} /$ rate $_{P F R}=1.75$. This ratio, considering the roughness of the physical model considered is 870 surprisingly in consistent with the ratio of the rates reported in Figure 11 (about 1.5).

This evidence suggests that the ISO reactor underestimate the rate by the limitation due to the mass transfer resistance. To work as a true PFR, the ISO reactor should 875 work at very high flow rates to minimize the mass transfer limitations which cause deviations from the ideal behavior of a PFR. However, in this way the conversion would be so low that the chemical analysis to assess the value of the conversion becomes problematic. In other words it is almost 880 impossible to obtain reliable results from the continuous reactor (PFR like) now used for the standardization.

\subsection{CST reactor size}

Practically, all the above opportunities (any volume, any shape, any flow) are limited by other issues. The mini- 885 mum flow is imposed: (a) by the detector if this is of sampling type. For example, commercially available chemiluminescence's detectors for $\mathrm{NO}_{\mathrm{x}}$ need an input flow ranging from 0.8 to $1.2 \mathrm{~L} \mathrm{~min}^{-1}$. Thus, using this configuration the minimum flow must be of the order $1.5-890$ 
2.0 $\mathrm{L} \mathrm{min}^{-1}$; (b) by the time required to clean, fill and wait to reach the steady state.

In the filling region, according to eq. [2], it is easy to demonstrate that after the reactor cleaning $\left(C_{\text {dark }}=0\right)$ the 895 concentration reaches the value $C(t) / C_{0}=1-a$, where $a$ is the error admitted as soon as $t_{a}$ is passed, when $\boldsymbol{x}>-\log$ $(a) / t_{a}$ and where $\boldsymbol{x}=F / V_{R}$. If $a$ relative error $a=0.001$ $(0.1 \%)$, and $t_{a}=3 \mathrm{~min}$ (to be fast) are considered, it results that $\boldsymbol{x}_{\min }=1$.

900 In the reaction region, according to eqs. [7] and [8] the decaying concentration after illumination reaches a value $C(t) / C_{\infty}=1+a$, where $a$ is the error admitted, when at least $t_{a}$ is passed, according to

$$
t_{a}=t_{R} \frac{\ln \left(\frac{D a}{a}\right)}{1+D a}=\frac{\ln \left(\frac{y}{a x}\right)}{x+y}
$$

905 where $\boldsymbol{x}=1 / t_{R}=F / V_{R}$ and $\boldsymbol{y}=D a / t_{R}=k_{R} S / V_{R}$ are the relevant normalized variables, with dimensions $\left[t^{-1}\right]$.

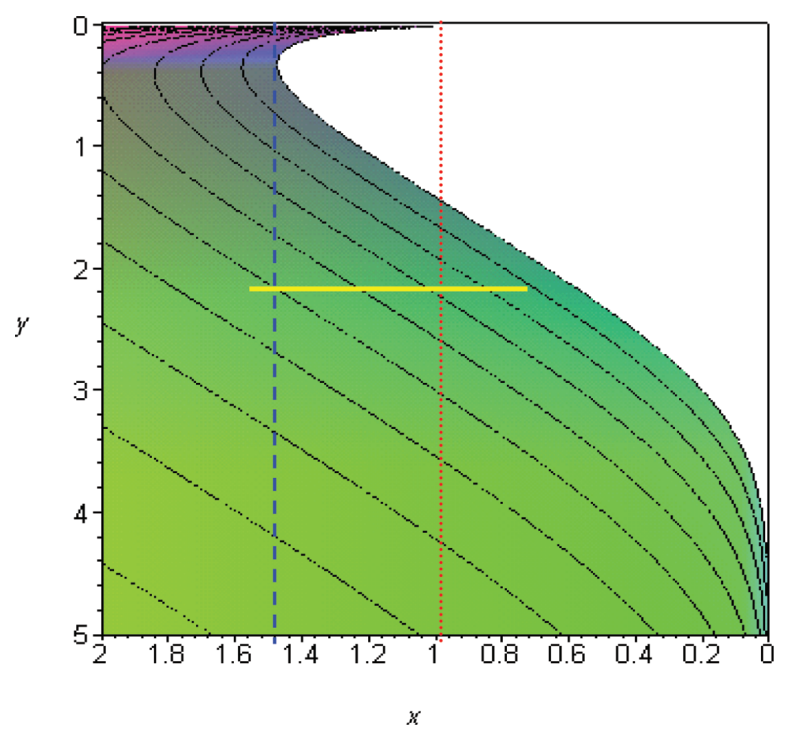

Figure 12 The colored region represents values of reciprocal time variables $\boldsymbol{x}=F / V_{R}$ and $\boldsymbol{y}=k_{R} S / V_{R}$ for which $C(t) / C_{\infty} \leq 1.001$ in less than three minutes. On the upper right corner: (white) values of $\boldsymbol{x}$ and $y$ which do not meet this requirement. For straight lines (see text): the dotted (red) line represents $x=1$, the condition for filling, the dashed (blue) line a risk free condition for $\boldsymbol{x}_{\min }$, the continuous (yellow) line the condition under which the experiments reported have been carried out.

The minimum time $t_{a}$ is definitely proportional to $t_{R}$. For a minimum flow required by the analyzer (say $1.2 \mathrm{~L} \mathrm{~min}^{-1}$ for a sampling device like the $\mathrm{NO}_{\mathrm{x}}$ analyzer), a minimum reason-

910 able volume $V_{R}$ to accommodate samples, say $1 \mathrm{~L}$, and larger volumes (say $10 \mathrm{~L}$ ) with a maximum flow of $5 \mathrm{~L} \mathrm{~min}^{-1}$ (as is the case when the flow is created with mass flow controllers), $\boldsymbol{x}$ can vary from $0.1(1 / 10)$ to 5 (say $5 / 1) \mathrm{min}^{-1}$.
A numerical analysis of eq. [22] is reported in Figure 12, where a maximum time to reach the steady 915 state with a relative error of $a=0.001$ was considered three minutes. The value of $\boldsymbol{y}$ can vary from 0 to infinity depending on the specific reactivity of the sample. As Figure 12 shows, to stay on the colored region where there is the certainty that the steady state is reached in 920 three minutes for any value of $\boldsymbol{y}, \boldsymbol{x}$ must be approximately $>1.5$ (see the dashed curve). This condition assures also that the time required to clean and fill the reactor are less than three minutes (see the value at $\boldsymbol{y}=0$, where according to above $\boldsymbol{x}_{\min }=1$ ). As a consequence, the minimum 925 value of $\boldsymbol{x}$ is about 1.5 for assuring that both filling and equilibrium value are reached within three minutes. This implies that the flow must be always larger than $3 / 2$ times the reactor volume. As the maximum flow could be $5 \mathrm{~L} \mathrm{~min}^{-1}$, a maximum reactor volume $V_{R, \max }=3.3 \mathrm{~L} 930$ could guaranty for any value of $\boldsymbol{y}$ a free risk operation with fast attainment of the steady state (three minutes).

Under the above conditions, after light ON the concentration reaches the steady state so rapidly (three minutes) that often the detector time response is slower, and the fit of 935 data using eq. [7] is useless. This is what happened in Figure 4, where all curves are superimposed just after illumination, because they are integrated over the time response of the detector. The data reported in Figure 4 confirm that the equilibration time is reached in less than three minutea 940 using a reactor with $V_{R}=2.85 \mathrm{~L}$ and flow ranging from 2 to $4.5 \mathrm{~L} \mathrm{~min}^{-1}$ ( $x$ ranging from 0.70 to 1.58). Since the actual equilibration time is less than three minutes, this implies that the actual value of $\boldsymbol{y}$ is far from the risky $\boldsymbol{y}=0.3$ (see Figure 12). From the data plotted as in Figure 9, an estimate 945 of $k_{R} S=6$ was figured out for exp.\#1, implying a minimum value of $y=6 / 2.85=2.1$. From Figure 12 (yellow line) the set used for $x$ and $y$ is well positioned in the colored region, far from the border with the white zone $\left(t_{a}>3 \mathrm{~min}\right)$.

Provided that the flow is always larger than $3 / 2$ of the 950 reactor volume, a range of values (e.g. $F=1.5-5 \mathrm{~L} \mathrm{~min}^{-1}$, $V_{R}=1.0-3.5 \mathrm{~L}$ ) rather than a fixed reactor volume and flow, are then suggested to be fixed in the standardized protocols. Larger reactors could also be used, again, provided that the flow is always larger than $3 / 2$ of the 955 reactor volume if a maximum time to reach the steady state would be quite short (three minutes). The settlement on larger equilibration times consequently leads to a different range of values for $F$ and $V_{R}$ that can be calculated using the reasoning reported above. Alternatively, 960 letting free the choice of reactor volume and flow, the standardized protocols must compel the certifying body to report the experimental run, demonstrating that the steady state is reached before and after illumination. 
965

\section{Conclusions}

From the above analysis, the following can be concluded:

(a) The CSTR configuration with a fan offers a lot of advantages for practical use, as any volume, any shape of catalyst and any flow of gas into the reactor can be used, provided that the time to reach stationary conditions conforms to the reactor volume. As the rate $\left(C_{o}\right)$ could vary with time for extra reasons (e.g. fast catalyst surface poisoning), a ratio $F / V_{R}$ as large as possible must be fixed.

975 (b) The CSTR configuration is superior to PFR as it is less dominated by resistance to mass transfer and, consequently, gives an evaluation of the photocatalytic rate more close to the actual surface one.

(c) The rate must be calculated using eq. [11]. In this 980 way it is equivalent to that estimated in a batch experiment.

(d) The rate calculation reported in the present norms for a flow-through experiment (see eq. [10]) underestimates the rate for a CSTR by a factor $1 /(1-\eta)$. The error is large when the conversion $\eta$ is large, that is when the flow is low or the sample surface is large, or the photocatalyst is particularly active. The use of eq. [10] with a PFR is theoretically incorrect.

(e) It is crucial that the flow-through experiment will be carried out at a conveniently measurable conversion $(0.2<\eta<0.8)$, where the experimental error is low, by adjusting the flow $F$, and then use the formula (11) to obtain $r\left(C_{0}\right)$.

(f) It is reasonable to recommend that in a flow-through experiment the conversion is monitored in continuum, if possible, and then $r\left(C_{0}\right)$ is calculated as function of time. In this way it will be possible to monitor the stability of the catalyst as a function of time. This last possibility is prevented by a batch or a recirculation experiment, because possible changes in catalyst activity are convoluted in the decaying curve of $C(t)$ vs. time. The evaluation of $k_{o b s}$ in eq. [4] will give an average value over the arbitrary time used for the experiment.

(g) The standardized protocols have to fix the counter 1005 pressure also exercised by vent tubes, by properly defining output reactor hole, tube diameter and length, otherwise the actual concentration inside the reactor is different from the nominal $C_{\mathrm{o}}$.

The above considerations, well supported by the experi- 1010 mental results here reported, could be suggested as a framework to develop protocols through which the rate evaluation by laboratories of research or by standardization bodies becomes comparable.

\section{List of symbols}

$\mu$

C

$c^{*}$

$C_{0}$

$\eta$

CSTR

$D a$

F

Io

$k_{\text {obs }}$

$k_{R}$

PFR

$r_{\infty}$

$r 0$

$R e$

$S$

$t^{\star}$

$t_{R}$

U

$V_{R}$

$\boldsymbol{X}$

$y$

\author{
kinematic viscosity of the fluid \\ concentration, inside the reactor for a CSTR and at \\ outflow \\ dimensionless normalized concentration $C^{\star}=C$ / \\ $c_{o}$ \\ input concentration of the pollutant in the reactor \\ conversion, defined as $=\left(1-C_{\text {out }}^{*}\right), \eta_{\infty}$ refers to \\ steady state \\ continuous stirred-tank reactor \\ dimensionless Damköhler number, $D a=k_{R} S / F$ \\ flow \\ light intensity reaching the surface of the sample \\ exponential decay constant for batch experiments, \\ $k_{\text {obs }}=k_{R} S / V_{R}$ \\ specific rate constant per unit area $S$ \\ plug flow reactor \\ photocatalytic rate calculated at steady state \\ photocatalytic rate per unit area, usually assumed \\ first-order $r(C)=k_{R} C=D a F C / S$ \\ Reynolds number \\ surface area of the sample exposed to light \\ dimensionless time $t^{\star}=t / t_{R}$ \\ reactor space time (residence time) $t_{R}=\left(V_{R} / F\right)$ \\ linear velocity of the fluid inside the reactor \\ reactor volume \\ reciprocal time variables $\boldsymbol{x}=F / V_{R}$ \\ reciprocal time variables $\boldsymbol{y}=k_{R} S / V_{R}$
}

\section{References}

1. COST Action 540 "PHONASUM", 2007-2010. Photocatalytic technologies and novel nanosurface materials. Available at: http://www.cost540.com/content/cost1.html

2. Minero C, Maurino V, Pelizzetti E. Mechanism of photocatalytic degradation of organic compounds. In: Schanze KS, photochemistry, Vol. 10, Chapter 6, Semiconductor photo-

3. Satuf ML, Brandi RJ, Cassano AE, Alfano OM. Ind Eng Chem Res 2005;44:6643-9.

4. Minero C. Catal Today 1999;54:205-16. chemistry and photophysics. New York: Marcel Dekker, 2003. 
6. ISO 22197-1. Fine ceramics (advanced ceramics, advanced technical ceramics) - Test method for air-purification performance of semiconducting photocatalytic materials - Part 1: Removal of nitric oxide, 2007.

7. UNI 11238-1. Determination of the catalytic degradation of organic micropollutants in air - Part 1: Photocatalytic cementitious materials, 2007.

8. Zhang J, Ayusawa T, Minagawa M, Kinugawa K, Yamashita H, Matsuoka M, et al. I Catal 2001;198:1-8.

9. AFNOR XP B44-013. Photocatalyse - méthode d'essais et d'analyses pour la mesure d'efficacité de systèmes photocatalytiques pour l'élimination des composés organiques volatils/odeurs dans l'air intérieur en recirculation - Test en enceinte confinée, December 2009.

10. Matsuda S, Hatano H. Powder Technol 2005;151:61-7.

11. Tatsuma T, Tachibana S-I, Fujishima A. I Phys Chem B 2001;105:6987-92.

12. AFNOR XP B44-011. Photocatalyse - méthode d'essai pour l'évaluation des matériaux photocatalytiques vis-à-vis de la dégradation des $\mathrm{NO}_{\mathrm{x}}$ - Méthode à un seul passage en mode tangentiel, 2009.

13. Ohko Y, Nakamura Y, Fukuda A, Matsuzawa S, Takeuchi K. J Phys Chem C 2008;112:10502-8.

14. ISO 22197-2. Fine ceramics (advanced ceramics, advanced technical ceramics) - Test method for air-purification performance of semiconducting photocatalytic materials - Part 2: Removal of acetaldehyde. 2011.

15. Negishi N, Matsuzawa S, Takeuchi K, Pichat P. Chem Mater 2007;19:3808-14.

16. ISO 22197-3. Fine ceramics (advanced ceramics, advanced technical ceramics) - Test method for air-purification performance of semiconducting photocatalytic materials - Part 3: Removal of toluene, 2011.
17. ISO/DIS 22197-4. Fine ceramics (advanced ceramics, 1070 advanced technical ceramics) - Test method for air-purification performance of semiconducting photocatalytic materials - Part 4: Removal of formaldehyde, Under development, TC206, stage 40.60 .

18. ISO/DIS 22197-5. Fine ceramics (advanced ceramics, 1075 advanced technical ceramics) - Test method for air-purification performance of semiconducting photocatalytic materials Part 5: Removal of methyl mercaptan, Under development, TC206, stage 40.60 .

19. Ibusuki T, Takeuchi K. J Mol Catal 1994;88:93-102.

20. Nakamura I, Sugihara S, Takeuchi K. Chem Lett 2000;29:1276-7.

21. Hashimoto K, Wasada K, Osaki M, Shono E, Adachi K, Toukai N, et al. Appl Catal B: Environ 2001;30:429-36.

22. Dalton JS, Janes PA, Jones NG, Nicholson JA, Hallam KR, Allen GC. Environ Pollut 2002;120:415-22.

23. Ichiura H, Kitaoka T, Tanaka H. Chemosphere 2003;51:855-60.

24. Devahasdin S, Fan C, Li JK, Chen DH. J Photochem Photobiol A: Chem 2003;156:161-70.

25. Lin YM, Tseng, YH, Huang, JH, Chao, CC, Chen CC, Wang I. Environ SciTechnol 2006;40:1616-21.

26. Wu JCS, Cheng Y-T. I Catal 2006;237:393-404.

27. Shelimov BN, Tolkachev NN, Tkachenko OP, Baeva GN, Klementiev KV, Stakheev AY, et al. J Photochem Photobiol A: Chem 2008;195:81-8.

28. Li L, Shen, Q, Cheng, J, Hao Z. Appl Catal B: Environ 1095 2010;93:259-66.

29. Cassano AE, Martín CA, Brandi RJ, Alfano OM. Ind Eng Chem Res 1996;34:2155-201.

30. Ballari MD, Alfano OM, Cassano AE. Chem Eng Sci 2010;65:4931-42.

31. Schlichting H, Gerster K. Boundary layer theory, 8th ed. Berlin: Springer, 2000:ISBN 3-540-66270-7. 